\title{
Observation of Non-Gaussian Conductance Fluctuations at Low Temperatures in Si:P(B) at the Metal-Insulator Transition
}

\author{
Swastik Kar, A. K. Raychaudhuri, and Arindam Ghosh* \\ Department of Physics, Indian Institute of Science, Bangalore 560 012, India \\ H. v. Löhneysen and G. Weiss \\ Physikalisches Institut, Universität Karlsruhe, D-76128 Karlsruhe, Germany
}

\begin{abstract}
We report investigations of conductance fluctuations (with $1 / f^{\alpha}$ power spectra) in doped silicon at low temperatures $(T<20 \mathrm{~K})$ as it is tuned through the metal-insulator transition (MIT) by changing the carrier concentration $n$. The scaled magnitude of noise, $\gamma_{\mathrm{H}}$, increases with decreasing $T$ following an approximate power law $\gamma_{\mathrm{H}} \sim T^{-\beta}$. At low $T, \gamma_{\mathrm{H}}$ diverges as $n$ decreases through the critical concentration $n_{\mathrm{c}}$, accompanied by a growth of low-frequency spectral weight. The second spectrum and probability density of the fluctuations show strong non-Gaussian behavior below $20 \mathrm{~K}$ as $n / n_{\mathrm{c}}$ decreases through 1 . This is interpreted as the onset of a glassy freezing of the electronic system across the MIT.
\end{abstract}

The Anderson-Mott metal-insulator transition (MIT) has been one of the most studied yet unresolved areas in condensed matter physics [1,2]. A fundamental issue of considerable interest is that of conductance fluctuation (noise) as $\sigma_{T=0} \rightarrow 0$. The two main aspects, at the approach of the transition, are the divergence of noise near the critical region and the onset of non-Gaussian fluctuations. Experimental investigations of the noise magnitude in thin films of $\operatorname{In}_{x} \mathrm{O}_{y}$ [3] at room temperature and in $\mathrm{Si}$ inversion layers [4] at low temperatures show a divergence near the MIT. The only investigation of non-Gaussian fluctuations at the MIT has been done in 2D silicon inversion layers [5]. In this Letter we present the results of the first extensive experimental investigations of nonGaussian conductance fluctuations in a bulk (3D) system (single crystals of P-doped $\mathrm{Si}$ ) as it is driven through the MIT at low temperatures. As $T \rightarrow 0$ and the system crosses over to the insulating regime, the noise magnitude diverges, with a sharp rise in the contribution of slow dynamics to the spectral power. The fluctuations in this regime become increasingly non-Gaussian.

The P-doped $\mathrm{Si}$ samples (in some cases Bcompensated) were taken from the same batches that were used earlier for transport studies near the MIT [6] with a range of doping levels $n$ from $3.3 \times 10^{18} / \mathrm{cm}^{3}$ to $1 \times 10^{19} / \mathrm{cm}^{3}$, covering both sides of the critical doping level $\left(n_{\mathrm{c}}\right)$ for MIT (see table, Fig. 1). Samples of length 300-500 $\mu \mathrm{m}$, width 50-100 $\mu \mathrm{m}$, and thickness 25-50 $\mu \mathrm{m}$ were bonded with $25 \mu \mathrm{m}$ gold wires to provide low-noise Ohmic contacts. Figure 1 shows the variation of conductivity $\sigma$ with $T$ down to $0.1 \mathrm{~K}$. When fitted to the simple relation $\sigma=\sigma_{0}+m T^{1 / 2}$, samples with $\sigma_{0}>0$ are taken as metallic. In the samples with $n / n_{\mathrm{c}} \leq 1$ the conductivity for $T<10 \mathrm{~K}$ follows Mott's variable range hopping with $\sigma(T)=\sigma_{c} \exp \left[-\left(T_{0} / T\right)^{1 / 4}\right] . \quad T_{0} \rightarrow 0$ as $n \rightarrow n_{\mathrm{c}}$ from the insulating side.
Noise was measured as a function of $T$ using a fiveprobe ac technique [7]. The time series of voltage fluctuations were digitally processed [with typically $(1-5) \times$ $10^{5}$ data points] to obtain the power spectra $S_{V}(f)$, which varied as $S_{V}(f) \sim 1 / f^{\alpha}$ in the range $10^{-3} \mathrm{~Hz} \leq f \leq 4 \mathrm{~Hz}$. The temperature was controlled to better than $\pm 1 \mathrm{mK}$. The spectra at $4.2 \mathrm{~K}$ were also taken by directly immersing the samples in liquid ${ }^{4} \mathrm{He}$ with the temperature stability better than $100 \mu \mathrm{K}$, and no significant difference was found.

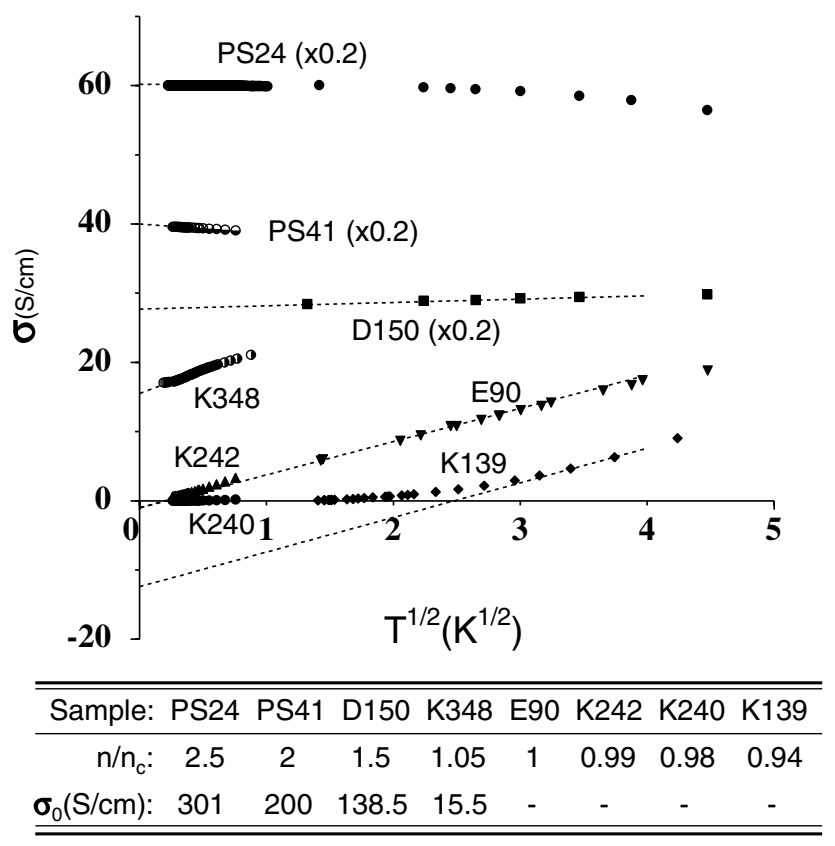

FIG. 1. Conductivity $\sigma$ vs temperature $T$ of the $\mathrm{Si}: \mathrm{P}(\mathrm{B})$ samples. $\sigma$ of the samples PS24, PS41, and D150 have been scaled down by a factor of 5 for convenience. The table shows the relevant sample properties. 
In all samples, $S_{V}(f)$ increases as the temperature is decreased below $100 \mathrm{~K}$. For $T>100 \mathrm{~K}$ the noise increases with $T$ again and this arises from classical mechanisms [8,9] which are outside the scope of this paper. Figure 2 shows a plot of the relative conductance fluctuation $\left\langle\Delta G^{2}\right\rangle / G^{2}=\int d f S_{V}(f) / V^{2}$, integrated over the experimental bandwidth, for a representative insulating sample (K139) with $n / n_{\mathrm{c}}=0.94$ for $T<10 \mathrm{~K}$. When measured with a sufficiently low biasing field, $\left\langle\Delta G^{2}\right\rangle / G^{2}$ varies approximately as $\sim A / T^{\beta}$. The behavior presented in Fig. 2 is typical for all samples on both sides of the MIT, right down to the lowest measured $T$.

As $T$ decreases, the measured noise becomes increasingly sensitive to the bias field $E$, and unless measured with a bias $E<E^{*}$, a characteristic field, the noise tends to "flatten out" or saturate as seen in Fig. 2. For $E>$ $E^{*}(T)$, the noise decreases according to an inverse power law with $E$. Any "sample heating" explains only a small part of this noise suppression. $E^{*}(T)$ in all the samples increases with increasing $T$. The results presented here correspond to $E<E^{*}$ such that $S_{V}(f) \propto V^{2}$.

Figure 3 shows a plot of the Hooge parameter [10] $\gamma_{\mathrm{H}}=f N S_{V}(f) / V^{2}$ at $1 \mathrm{~Hz}$ as a function of $n / n_{\mathrm{c}}$ for different $T, N$ being the total number of carriers in the measured volume. For $n / n_{\mathrm{c}}>1, \gamma_{\mathrm{H}}$ is comparatively small but as $n / n_{\mathrm{c}} \rightarrow 1, \gamma_{\mathrm{H}}$ increases slowly. At lower temperatures $(T<6 \mathrm{~K})$ close to $n / n_{\mathrm{c}}=1, \gamma_{\mathrm{H}}$ starts increasing rapidly and changes drastically over several orders of magnitude as it goes over to the insulating side. The strong temperature dependence of $\gamma_{\mathrm{H}}$ in the immediate vicinity of $n_{\mathrm{c}}$ is shown in inset (a) of Fig. 3 .

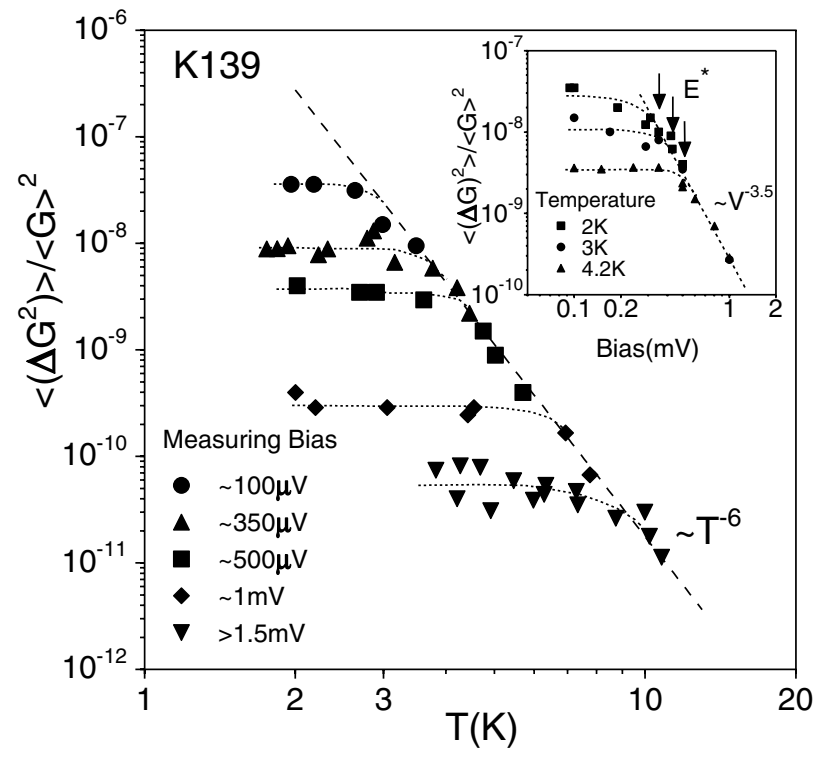

FIG. 2. $\left\langle\Delta G^{2}\right\rangle / G^{2}$ vs $T$ in K139 measured at different biasing voltages, showing a $T^{-\beta}$ dependence of noise at the lowest measuring bias. The inset shows how $\left\langle\Delta G^{2}\right\rangle$ deviates from a $G^{2}$ dependence at larger measuring biases.
At higher temperatures ( $T \geq 6 \mathrm{~K}$ ), the value of $\gamma_{\mathrm{H}}$ passes through a sharp peak at a value of $n / n_{\mathrm{c}}$ very close to but less than 1 [11].

Figure 3 inset (b) shows the variation of $\beta$ as a function of $n / n_{\mathrm{c}}$. For $n \gg n_{\mathrm{c}}, \beta \approx 0.5$ as expected from universal conductance fluctuation (UCF) $[12,13]$, slowly increasing to 1 as $n$ approaches $n_{\mathrm{c}}$. This can also be rationalized within the framework of UCF when the conductance becomes scale invariant [14]. However, $\beta$ shows a rather sharp increase while it passes through the MIT and becomes $\gg 1$ in the most insulating sample. The changes in $\gamma_{\mathrm{H}}$ and $\beta$ across the MIT are extremely large and could not be analyzed within the framework of UCF (which is saturated in these systems [12]) or kinetic models [10].

On the weakly localized metallic side $\left(n / n_{\mathrm{c}} \geq 2\right)$ the measured noise is Gaussian. However, at low temperature $(T=4.2 \mathrm{~K})$ the noise departs strongly from a Gaussian as $n / n_{\mathrm{c}} \rightarrow 1$, as seen from the second spectrum [15] defined as $S^{(2)}(f)=\int_{0}^{\infty}\left\langle V^{2}(t) V^{2}(t+\tau)\right\rangle_{t} \cos (2 \pi f \tau) d \tau$. For uncorrelated fluctuations, $S^{(2)}(f)$ [16] has a frequency independent "white" spectrum (Gaussian background). A departure of $S^{(2)}(f)$ from a white spectrum will be an indication of non-Gaussian fluctuations. Figure 4(a) shows the second spectra $S^{(2)}(f)$ (with the Gaussian background subtracted) of five uncompensated samples close to the MIT at $T=4.2 \mathrm{~K}$, normalized by $\left[\int_{f_{L}=1 \mathrm{~Hz}}^{f_{H}=2 \mathrm{~Hz}} S_{V}(f) d f\right]^{2}$. The spectra $(f<1 \mathrm{~Hz})$ fit the relation $S^{(2)}(f) \propto f^{-p}$, which is usually taken as a sign of interaction between the fluctuators [15]. As a function of $n / n_{\mathrm{c}}, p$ changes [see inset, Fig. 4(a)] from a small value



FIG. 3. The scaled noise $\gamma_{\mathrm{H}}$ for different values of $n / n_{\mathrm{c}}$. Inset (a) shows the same graph, magnified around $n / n_{\mathrm{c}}=1$. Inset (b) shows the $n / n_{\mathrm{c}}$ dependence of $\beta$. 


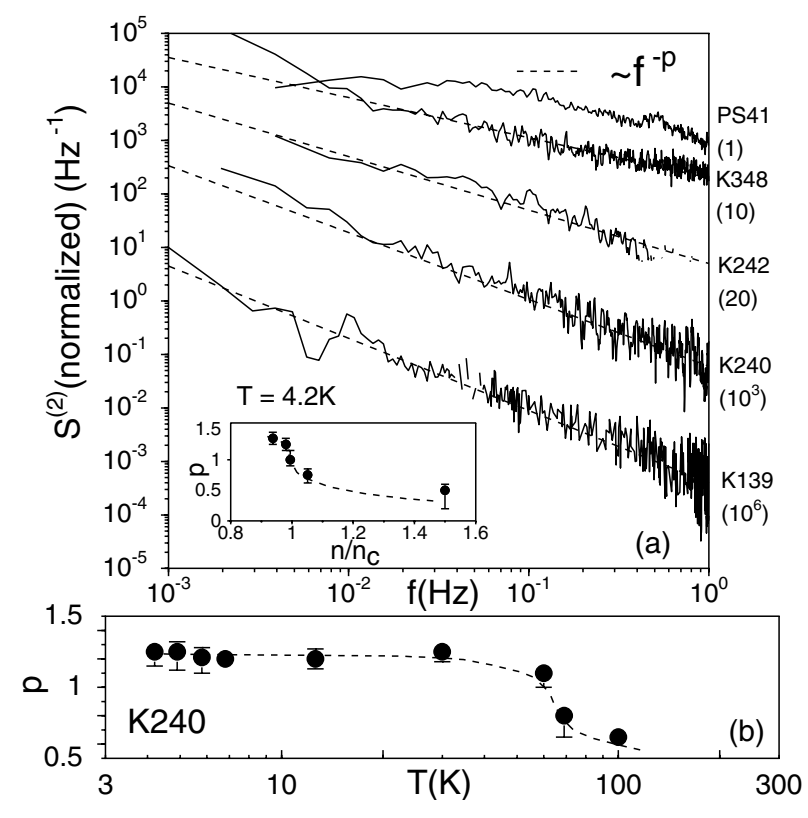

FIG. 4. (a) Normalized second spectrum $S^{(2)}(f)$ at $4.2 \mathrm{~K}$. The spectra have been shifted down vertically for visual clarity by factors shown in brackets. The inset shows the sharp growth of frequency dependence at the critical concentration $n=n_{\mathrm{c}}$ at $4.2 \mathrm{~K}$. (b) $T$ dependence of the exponent $p$ (see text) in the sample K240.

for $n / n_{\mathrm{c}}>1$, to $\sim 1.5$ as the insulating state is reached, changing rapidly at $n=n_{\mathrm{c}}$. Figure 4(b) shows how $p$ varies from a small value $<0.5$ to a large value $\sim 1.3$ in the insulating sample K240 as the temperature is lowered. These observations clearly show that as the MIT is approached the dynamics that gives rise to the conductance fluctuation becomes increasingly non-Gaussian and this is more so as the temperature is lowered.

The deviation from Gaussianity can also be seen through the fluctuation probability density function (PDF). This is the probability $P(|\Delta V|)$ of occurrence of a voltage jump of magnitude $|\Delta V|$ due to the fluctuating conductance. Figure 5 shows representative data plotted as $P(|\Delta V|)$ vs $\left|\Delta V^{2}\right|$. The upper panel shows that while the metallic sample PS41 has a Gaussian PDF at $4.2 \mathrm{~K}$, the insulating samples K242 and K139 exhibit deviations from a Gaussian for higher values of $|\Delta V|$. The deviation grows as $n$ decreases below $n / n_{\mathrm{c}}$ as well as with decreas$\operatorname{ing} T$, as seen in $\mathrm{K} 240$, lower panel of Fig. 5. We find that the non-Gaussian tails can be expressed with a lognormal dependence on $|\Delta V|$, where $\ln P(|\Delta V|) \propto$ $(\ln |\Delta V|)^{2}$, as predicted by several theoretical investigations in this regime [17].

The divergence of the conductance fluctuations at the MIT has been predicted in some models [18]. However, it is unlikely to arise from the usual dynamics of atomic defect relaxations $[10,13,15]$ as the strongest coupling mechanism that can produce conductance fluctuation from atomic dynamics is UCF which itself saturates at a variance of $e^{2} / h$. The measured noise for $n \simeq n_{\mathrm{c}}$ is much larger than that estimated from the UCF theory. Further, there is no reason why the defect dynamics should become more correlated as $n$ is decreased.

There is a marked increase in slow relaxations as $n$ is decreased. Figure 6(a) shows a segment of the relative fluctuations $\Delta R(t) /\langle R\rangle$ at $T=4.2 \mathrm{~K}$ in real time for two representative samples, $\mathrm{K} 348\left(n / n_{\mathrm{c}}=1.05\right)$ and $\mathrm{K} 139$ $\left(n / n_{\mathrm{c}}=0.94\right)$. The fluctuation is much larger as well as slower in insulating K139. The slow fluctuation seen in K139 was typical of the insulating samples and grew with decrease in $n$. The corresponding power spectra [Fig. 6(b) inset] of these time series had a $1 / f^{\alpha}$ behavior. $\alpha \sim 0.75$ in the metallic limit and rises sharply across the transition to reach a value $\sim 1.25$ in the insulating regime [Fig. 6(b)], indicating a distinctly enhanced contribution of slow fluctuations.

Inhomogeneous systems near a percolation threshold may give rise to non-Gaussian fluctuations, even for independent fluctuators, as proposed in the dynamical current redistribution (DCR) model [19], which usually

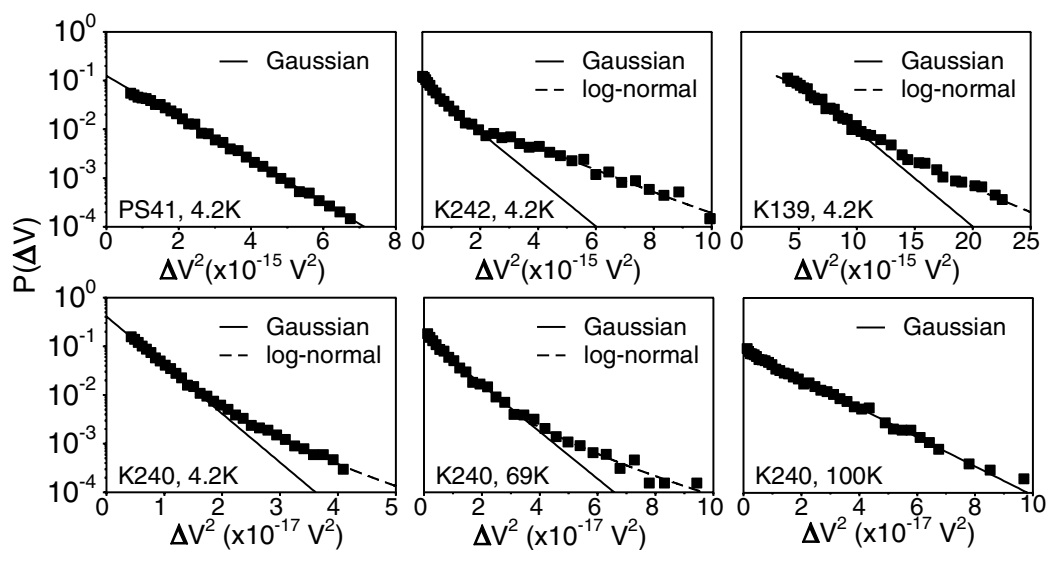

FIG. 5. Upper three panels show the PDF at 4.2 K for the metallic sample PS41, and insulating samples K242 and K139, which develop a non-Gaussian tail. The lower panels show how this tail grows for another insulating sample K240 with decreasing $T$. 

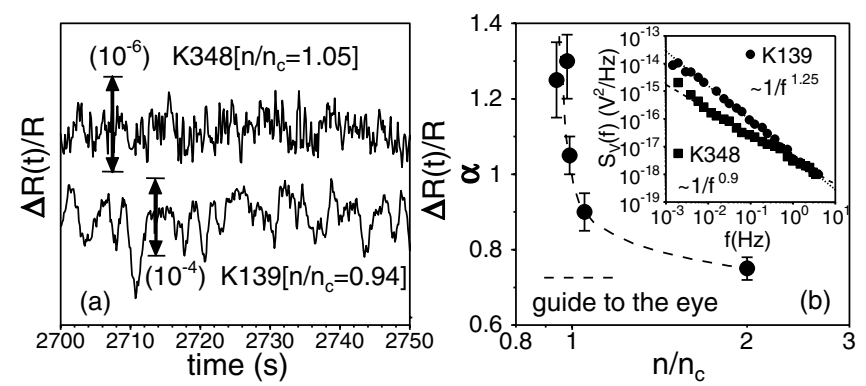

FIG. 6. (a) Time series of $\Delta R(t) /\langle R\rangle$ for K348 (Gaussian fluctuations) and K139 (non-Gaussian, with random metastable states). (b) Variation of $\alpha$ (see text) with $n / n_{\mathrm{c}}$. The inset shows the power spectra of the two time traces in (a).

works well in systems with large local fluctuations. With the fluctuations $(\Delta R / R)$ in our most insulating sample, K139, being $<10^{-4}$ [Fig. 6(a)], the applicability of this model is questionable in our system. Moreover, DCR fluctuators appear in series, tending to decrease $\alpha$. In contrast, we find a sharp increase in slow dynamics and an enhanced $\alpha$. This indicates a growth in correlations within the fluctuators, leading to large, slow fluctuations. We suggest that this is an indication of a glasslike freezing similar to what has been seen in spin glasses [15]. Analysis of conductivity data close to the MIT has shown that a comprehensive scaling description could be obtained if a glasslike transition is invoked [20]. Recent experiments on 2D Si inversion layers [4,5] show divergingly large low-frequency non-Gaussian fluctuations near the transition, interpreted as glasslike freezing of the electrons [21]. The similarity of the non-Gaussian spectra seen in both 2D and 3D (present investigation) raises the interesting possibility that such correlated dynamics may indeed be a universal feature of the MIT irrespective of dimensionality. At the MIT the single particle density of states can show both temporal and spatial fluctuations and if the dynamics is glassy then the relaxation time becomes an exponential function of the correlation length [20]. In this regime, the fluctuations are likely to be electronic in origin. The slowing down of the time scale of charge fluctuations is due to correlated transitions of electrons over a large number of metastable states and is in agreement with simulational results [22]. We propose that the large rise in the low-frequency noise at the approach to transition is a manifestation of lowfrequency dynamics of the fluctuations in the local density of states which are strongly correlated by Coulomb interaction in the absence of strong screening.

This work was supported by CSIR, India, DFG, Germany (SFB 195), and a DST-DAAD exchange collaboration.
*Present address: Cavendish Laboratory, University of Cambridge, Madingley Road, Cambridge, United Kingdom.

[1] P.W. Anderson, Phys. Rev. 109, 1492 (1958); P. A. Lee and T.V. Ramakrishnan, Rev. Mod. Phys. 57, 287 (1985).

[2] D. Belitz and T. R. Kirkpatrick, Rev. Mod. Phys. 66, 261 (1994); E. Abrahams et al., Rev. Mod. Phys. 73, 251 (2001); H. v. Löhneysen, Philos. Trans. R. Soc. London, Ser. A 356, 139 (1998).

[3] O. Cohen and Z. Ovadyahu, Phys. Rev. B 50, 10442 (1994); R. E. Johanson and S. O. Kasap, J. Vac. Sci. Technol. A 20, 1027 (2002).

[4] S. Bogdanovich and D. Popović, Phys. Rev. Lett. 88, 236401 (2002).

[5] J. Jaroszyński et al., Phys. Rev. Lett. 89, 276401 (2002).

[6] We have used $n_{c}=3.52 \times 10^{18} / \mathrm{cm}^{3}$ for the uncompensated samples and $n_{\mathrm{c}} \approx 4.5 \times 10^{18} / \mathrm{cm}^{3}$ for the Bcompensated samples: E90 with $60 \%$ compensation and D150 with $40 \%$ compensation. U. Thomanschefsky and D. F. Holcomb, Phys. Rev. B 45, 13356 (1992); H. Stupp et al., Phys. Rev. Lett. 71, 2634 (1993).

[7] J. H. Scoffield, Rev. Sci. Instrum. 58, 985 (1987).

[8] A. K. Raychaudhuri et al., Pramana - Journal of Physics 58, 343 (2002).

[9] S. Kar and A. K. Raychaudhuri, J. Phys. D 34, 3197 (2001).

[10] P. Dutta and P. M. Horn, Rev. Mod. Phys. 53, 497 (1981).

[11] A. Ghosh and A. K. Raychaudhuri, J. Phys. Condens. Matter 11, L457 (1999). Our previous attempts to observe a large change in $\gamma_{\mathrm{H}}$ at the transition were somewhat inconclusive because we did not go deep into the insulating state and we did not take into account the dependence on the measuring bias.

[12] A. Ghosh and A. K. Raychaudhuri, Phys. Rev. Lett. 84, 4681 (2000).

[13] P. A. Lee, A. D. Stone, and H. Fukuyama, Phys. Rev. B 35, 1039 (1987); J. S. Moon, N. O. Birge, and B. Golding, Phys. Rev. B 56, 15124 (1997).

[14] A. Ghosh and A. K. Raychaudhuri, in Proceedings of the 16th International Conference on Noise in Physical Systems and $1 / f$ Fluctuations (World Scientific, Singapore, 2001), p. 107.

[15] M. B. Weissman, Rev. Mod. Phys. 60, 537 (1988).

[16] G. T. Seidler and S. A. Solin, Phys. Rev. B 53, 9753 (1996).

[17] B. L. Altshuler et al., JETP Lett. 43, 441 (1986); Phys. Lett. 134, 488 (1989).

[18] M. C.W. van Rossum et al., Phys. Rev. B 55, 4710 (1997); B. Shapiro, Phys. Rev. Lett. 65, 1510 (1990).

[19] G. T. Seidler et al., Phys. Rev. Lett. 76, 3049 (1996).

[20] J. H. Davies et al., Phys. Rev. Lett. 49, 758 (1982); D. Belitz and T. R. Kirkpatrick, Phys. Rev. B 52, 13922 (1995); A.Vaknin et al., Phys. Rev. Lett. 81, 669 (1998).

[21] A. A. Pastor and V. Dobrosavljević, Phys. Rev. Lett. 83, 4642 (1999); V. Dobrosavljević et al., Phys. Rev. Lett. 90, 016402 (2003).

[22] Sh. Kogan, Phys. Rev. B 57, 9736 (1998). 